\title{
ANÁLISE MULTICRITERIAL DA ESTABILIDADE ECOLÓGICA EM TRÊS MODELOS DE RESTAURAÇÃO FLORESTAL
}

\section{Gustavo Galetti®}

Mestre em Planejamento e Uso de Recursos Renováveis, Universidade Federal de São Carlos (UFSCar) Sorocaba (SP), Brasil.

\section{José Mauro Santana da Silva(i)}

Professor adjunto do Centro de Ciências e Tecnologias para Sustentabilidade (CCTS) do Departamento de Ciências Ambientais (DCA) da UFSCar Sorocaba (SP), Brasil.

\section{Fatima Conceição}

Márquez Piña-Rodrigues(i)

Professora titular do CCTS do DCA da UFSCar - Sorocaba (SP), Brasil.

\section{Ivonir Piotrowiski@}

Doutorando em Planejamento e Uso de Recursos Renováveis, UFSCar - Sorocaba (SP), Brasil.

\section{Endereço para correspondência:} Gustavo Galetti - Rua Coronel Manoel Leme, 2.037- Novo Jardim Belém - CEP 13690-000Descalvado (SP), Brasil-E-mail: gustavo_galetti@hotmail.com

Recebido em: 17/10/2017

Aceito em: 04/05/2018

\section{RESUMO}

Tendo em vista a crescente necessidade de novos modelos para restauração florestal, o presente estudo teve o objetivo de avaliar se alta diversidade de espécies vegetais, funções e densidade em restauração florestal resultaram na recuperação da estabilidade do sistema, e quais as condições ou processos que afetaram o restabelecimento da funcionalidade ecológica nos sistemas avaliados. Para isso, um protocolo de indicadores de restauração foi aplicado em uma área de referência e em três áreas de restauração, em floresta estacional semidecidual, uma delas no modelo denso-diversofuncional ( 3 mudas por $\mathrm{m}^{2}$ ), com 53 meses de idade; e duas no modelo de preenchimento e diversidade (espaçamento $3 \times 2 \mathrm{~m}$ ), com 35 e 60 meses de idade. Os resultados indicam que o modelo denso-diverso-funcional é eficiente na restauração da funcionalidade ecológica já aos 53 meses e difere do sistema preenchimento e diversidade em relação às condições necessárias para promover a proteção do solo e o aporte de serapilheira.

Palavras-chave: processos ecológicos; indicadores ambientais; recuperação de áreas degradadas.

\section{ABSTRACT}

Considering the growing need for new models for forest restoration, the present study had the objective of evaluating the high diversity of plant species, functions and density in forest restoration, resulting in the recovery of the system's stability, and the conditions or processes that affected the restoration of ecological functionality in the systems evaluated. For this, a restoration indicator protocol was applied in a reference area and in three restoration areas, in a semideciduous seasonal forest, a dense-diversefunctional model ( 3 seedlings per $\mathrm{m}^{2}$ ), with 53 months of age and two in the model of fill and diversity (spacing $3 \times 2 \mathrm{~m}$ ), with 35 and 60 months of age, respectively. The results indicate that the dense-diverse-functional model is efficient in the restoration of the ecological functionality at 53 months and differs from the system fill and diversity in relation to the conditions needed to promote soil protection and litter loading.

Keywords: ecological processes; environmental indicators; recovery of degraded areas. 


\section{INTRODUÇÃO}

Devido à pressão antrópica, decorrente principalmente da expansão urbana e de atividades agropecuárias, grande parte das regiões tropicais apresenta sua cobertura florestal nativa altamente fragmentada e/ou restrita a pequenas porções de terra, resultando em crescente perda de biodiversidade (DEAN, 1996). No Brasil, estima-se o déficit de 43 milhões de hectares de áreas de preservação permanente e 42 milhões de hectares de reserva legal, sendo um desafio a restauração ambiental dessas áreas (SOARES-FILHO et al., 2014).

O processo de restauração de áreas degradadas pode ser entendido como a reconstrução dos processos ecológicos, garantindo a perpetuação e a evolução da comunidade no espaço e no tempo, contando com recursos bióticos suficientes para continuar seu desenvolvimento sem mais assistência ou subsídio. Um ecossistema restaurado vai se sustentar sozinho estrutural e funcionalmente, mostrando estabilidade às faixas normais de variação de estresse e perturbação ambiental, além de interagir com ecossistemas contíguos por meio de fluxos bióticos e abióticos (SER; POLICY WORKING GROUP, 2004; WRIGHT et al., 2009; ENGEL, 2011; SUGANUMA; DURIGAN, 2015). Nessa perspectiva, a estabilidade é um processo adimensional que reflete a capacidade de o sistema manter sua trajetória apesar do estresse ambiental, atingido parcialmente com base na capacidade do ecossistema para resistência e resiliência (DÍAZ; CABIDO, 2001; ENGEL; PARROTA, 2003; SER; POLICY WORKING GROUP, 2004; BASTIAN, 2013).

Resistência é o termo que descreve a habilidade do ecossistema manter seus atributos estruturais e funcionais quando sob condições de estresse e perturbações, ou seja, é a capacidade de o sistema suportar variações quando submetido a distúrbios ambientais. Já a resiliência é a capacidade de recuperar os atributos estruturais e funcionais que sofreram danos por estresse ou perturbação (DÍAZ; CABIDO, 2001; SER; POLICY WORKING GROUP, 2004; OLIVER et al., 2016); medida em função do tempo que o sistema demora para retornar à situação de equilíbrio dinâmico (ENGEL \& PARROTTA, 2003; SCHEFFER, 2015).

A disseminação de modelos pré-estabelecidos de restauração em detrimento de ajustes a cada condição tem sido apontada como uma das causas do insucesso nos plantios de restauração (DURIGAN et al., 2010).
Confirmando isso, levantamentos realizados em São Paulo mostraram que menos de $25 \%$ das áreas restauradas nos últimos 10 a 20 anos, para atender medidas legais, estavam parcialmente estabelecidas; e as demais não tiveram sucesso (RODRIGUES, 2013; FERNANDES; FREITAS; PIÑA-RODRIGUES, 2017). Esse debate se torna relevante pelas mudanças dos paradigmas na restauração, que levam a enfatizar não o produto da sucessão, mas sim os processos internos como as interações bióticas e abióticas e as externas - interações com a paisagem - , que levam ao restabelecimento da trajetória sucessional (SUDING; GROSS, 2006).

Por outro lado, discute-se a restauração e sua relação direta com a retomada de processos ecológicos diretamente relacionados ao aumento da riqueza e diversidade de espécies, fato que norteou vários mecanismos legais como a Resolução da Secretaria de Meio Ambiente (SMA) no 08/2008 (SÃO PAULO, 2008) em São Paulo. Contudo, a riqueza de espécies na restauração não necessariamente proporciona ocorrência de interações geradoras de processos ecológicos, uma vez que espécies distintas podem exercer funções similares, reduzindo a diversidade funcional presente no ecossistema (PETCHEY et al., 2004; FORRESTER; BAUHUS, 2016). A diversidade funcional considera que diferenças ecológicas, fisiológicas e morfológicas entre as espécies resultam em diferenças de funções das mesmas dentro de um ecossistema (PETCHEY; GASTON, 2006). Assim, os processos em ecossistemas funcionalmente diversos ocorrerão em detrimento dos funcionalmente redundantes, por conta da diversidade de características funcionais (NAEEM, 1998; CIANCIARUSO; SILVA; BATALHA, 2009; TRINDADE FILHO; LOYOLA, 2010). A relação da diversidade funcional com a riqueza de espécies é complexa e depende do contexto no qual as espécies se encontram (CADOTTE; CARSCADDEN; MIROTCHNICK, 2011). No entanto, a diversidade funcional afeta a comunidade e exerce importante papel na restauração ecológica (NAEEM, 2002; CADOTTE; CARSCADDEN; MIROTCHNICK, 2011).

Como a diversidade funcional é geradora de processos ecológicos, torna-se de extrema importância o ajuste das metodologias para restauração florestal envolvendo o conhecimento do local, a seleção correta da metodologia de plantio, a biologia e a forma de utilização das espécies para o sucesso do plantio heterogêneo (RODRIGUES; BRANCALION; ISERNHAGEN, 
2009; BRANCALION et al., 2010). Para isso, devem ser consideradas não apenas as espécies a serem plantadas, mas principalmente suas funções no sistema (CADOTTE; CARSCADDEN; MIROTCHNICK, 2011).

Evidências empíricas têm demostrado que a resposta ecossistêmica ao aumento na riqueza de espécies é variável, podendo ter diferentes magnitudes, dependendo do grau de dominância das espécies perdidas ou adquiridas, da força de suas interações com outras espécies, da ordem em que as espécies são perdidas, dos traços funcionais das espécies, da complementariedade de caracteres funcionais das espécies e das interações bióticas e abióticas que regulam a magnitude e a expressão desses nas funções ecossistêmicas (DÍAZ \& CABIDO, 2001; NAEEM, 2002; HOOPER et al., 2005). De fato, mais de 50 padrões de potenciais de respostas ecossistêmicas já foram descritos (LOREAU, 1998; NAEEM, 2002), sendo as relações mais comuns a relação linear positiva, a assintótica e a ausência de relação óbvia (ENGEL, 2011).

Métodos de restauração comumente adotados, como plantio em área total, enriquecimento, adensamento ou nucleação, consideram o emprego da diversidade a partir do uso de grupos funcionais de plantio, dividindo-os em "espécies de preenchimento" (RODRIGUES; BRANCALION; ISERNHAGEN, 2009) ou "espécies de recobrimento", que recobrem rapidamente o solo (geralmente espécies pioneiras ou secundárias iniciais), e "espécies de diversidade", que crescem ou recobrem o solo mais lentamente, mas aumentam a diversidade por pertencerem a diversos grupos sucessionais (GANDOLFI; BELOTTO; RODRIGUES., 2009). Outros modelos de plantios de restauração vêm sendo utilizados no Brasil e em vários países, tendo como premissa a associação de alta diversidade com as funções ecológicas e a riqueza de espécies (MIYAWAKI, 1998; MIYAWAKI \& ABE, 2004; LELES et al., 2011). Dentre eles, destacam-se os métodos Myawaki (SCHIRONE; SALIS; VESSELLA, 2011) e o denso-diverso-funcional (DDF), que associam alta riqueza de espécies e de funcionalidade ecológica à alta densidade de plantas, promovendo rápida cobertura das áreas visando acelerar o reestabelecimento de processos como a sucessão e a ciclagem de nutrientes, entre outros (PIÑA-RODRIGUES; REIS; MARQUES, 1997).

Diante da complexa relação entre a riqueza de espécies e o funcionamento de ecossistemas, surge a necessidade de avaliar como a riqueza e a diversidade de espécies podem ser manipuladas e como o funcionamento do ecossistema deve ser medido como uma variável resposta a essas manipulações, fornecendo subsídios e referenciais teóricos para que as discussões sobre o tema continuem evoluindo (NAEEM, 2002; CARDINALE et al., 2009; LAUGHLIN, 2014; TILMAN; ISBELL; COWLES, 2014).

\section{OBJETIVOS}

Considerando o exposto, o presente trabalho pretende responder às seguintes questões:

- Sistemas de restauração densos e biodiversos foram eficientes na restauração da estabilidade, re- siliência, confiabilidade e processos ecológicos em relação a outros modelos?;

- Quais as condições ou processos que afetaram o estabelecimento da funcionalidade ecológica nos modelos de restauração avaliados?

\section{MATERIAL E MÉTODOS}

\section{Áreas de estudo}

Em zonas de floresta estacional semidecidual, na Bacia Hidrográfica do Rio Sorocaba e Médio Tietê, estado de São Paulo, foram avaliadas quatro áreas com diferentes modelos e idades de restauração. A região apresenta clima tropical de altitude com verão moderadamente quente, temperatura média anual de $22^{\circ} \mathrm{C}$, com períodos secos nos meses de junho a agosto, precipitação inferior a $100 \mathrm{~mm}$ mensais e temperatura mínima de $15^{\circ} \mathrm{C}$ e máxi- ma de $27^{\circ} \mathrm{C}$ (INMET, 2015). O relevo da região encontra-se em área de transição entre o planalto atlântico e a depressão periférica paulista, as altimetrias predominantes estão entre 600 e $650 \mathrm{~m}$, enquanto as declividades variam entre 5 e 10\% (ROSS; MOROZ, 1997). A unidade pedológica presente é de Latossolos Vermelhos, constituído por Latossolos Roxos e Latossolos Vermelho-Escuros distróficos, segundo o Mapa Geomorfológico de Rossi (2017). 
Como área de referência (AR) foi selecionado um fragmento de 4 ha situado a $\left(23^{\circ} 34^{\prime} 57^{\prime \prime}\right.$ S; $23^{\circ} 35^{\prime} 25^{\prime \prime} \mathrm{S}$ e $47^{\circ} 31^{\prime} 50^{\prime \prime} \mathrm{W} ; 47^{\circ} 31^{\prime} 00^{\prime \prime} \mathrm{W}$ ), na mesma região das restaurações. A área encontra-se em estágio intermediário de sucessão, apresenta regeneração com elevada riqueza de espécies transitórias, formada em sua maioria por espécies não pioneiras e zoocóricas, com dominância de Aspidosperma olivaceum, Cordia trichotoma, Ocotea elegans, Guarea guidonia, Psychotria carthagenensis, Esenbeckia leiocarpa e Casearia obliqua (KORTZ et al., 2014).

Como áreas de restauração foram analisados:

- Um plantio de 4 ha implantado em 2011, no modelo DDF, com idade de 53 meses, onde foram plantadas 81.594 mudas com 3 indivíduos $/ \mathrm{m}^{2}$, de 142 espécies, sendo $47 \%$ pioneiras e $53 \%$ não pioneiras, selecionadas de acordo com Miyawaki (1999), considerando as suas funções ecológicas no sistema (atração da fauna, aporte de biomassa, interação com microrganismos do solo, cobertura do solo);

- Duas áreas plantadas de diferentes idades (35 e 60 meses) em espaçamento $3 \times 2 \mathrm{~m}$ no modelo preenchimento e diversidade (P\&D) de acordo com Rodrigues, Brancalion e Isernhagen (2009), com proporção aproximada de $60 \%$ de pioneiras e $40 \%$ de não pioneiras, de acordo com a Resolução SMA no 08/2008 (SÃO PAULO, 2008). Na área de 35 meses (P\&D-35), foram plantados 20 ha com 97 espécies distribuídas em 33 mil mudas. Na área de 60 meses de idade (P\&D-60) foram plantados 37,25 ha com 80 espécies divididas em 62 mil mudas.

As coletas em campo foram realizadas entre os meses de janeiro e maio de 2016, em 56 parcelas de $10 \times 10$ m, sen-

\section{Avaliação da funcionalidade ecológica}

O método "Marco para la Evaluación de Sistemas de Manejo de Recursos Naturales Incorporando Indicadores de Sustentabilidad" (MESMIS) auxilia na escolha de indicadores, pois considera o comportamento das características mais latentes do sistema avaliado e como essas podem levá-lo a atingir estabilidade e resiliência (MASERA; ASTIER; LÓPEZ-RIDAURA, 1999). O método propicia a criação de protocolos de seleção e aplicação de indicadores, que consideram atributos, descritores e a integridade ecológica do ecossistema, com isso, permitindo a caracterização dos sistemas em diferentes dimensões ecológicas. Os da- do 6 parcelas em AR, 15 no DDF, 15 no P\&D-60 e 20 no P\&D-35. Para os descritores de diversidade e parâmetros funcionais da comunidade, todos os indivíduos arbóreos foram identificados, e mensuradas sua altura total $(\mathrm{cm})$ e circunferência à altura do colo (CAC) (mm). Cada espécie foi classificada por grupo sucessional (pioneira e não pioneira), empregando-se os mesmos critérios da Resolução SMA no 08 (SÃO PAULO, 2008). Os indivíduos arbóreos observados foram avaliados quanto à presença de epífitas, cipós e lianas. No descritor de controle e manejo foram estimados a cobertura do solo por copa, calculada segundo as indicações da Resolução SMA no 32/2014 (SÃO PAULO, 2014); incidência de luz (\%); cobertura do solo (\%) com gramíneas invasoras; e avaliação de impactos causados pela presença humana, tanto positiva (manejo, capina, ausência de incêndios) quanto negativa (trilhas, caminhos e incêndios), realizada por meio de inspeção visual das parcelas de $100 \mathrm{~m}^{2}$ e com base em informações obtidas com os responsáveis pela manutenção. No descritor de proteção do solo e aporte de serapilheira foram estimados: cobertura do solo (\%) com herbáceas e serapilheira e altura da serapilheira $(\mathrm{cm})$. Os indicadores de cobertura do solo com serapilheira, herbáceas e gramíneas foram obtidos com o uso de um quadro de $0,50 \times 0,50 \mathrm{~m}$, subdividido em 4 quadrículas de $0,25 \times 0,25 \mathrm{~m}$, lançado em 3 pontos de cada parcela. Cada quadrícula preenchida em mais da metade de sua área representou $25 \%$ de cobertura. Posteriormente, foram calculadas as médias de porcentagem de cobertura de cada indicador. Nesses mesmos pontos foi determinada a incidência de luz com um luxímetro (Klux) posicionado a $1 \mathrm{~m}$ do solo na direção norte; e também foi medida a incidência de luz a céu aberto a $1 \mathrm{~m}$ do solo a cada 30 min durante as coletas, para posterior cálculo da porcentagem de incidência de luz dentro das parcelas.

dos obtidos por meio desses indicadores auxiliam a detectar pontos críticos no funcionamento dos ecossistemas, a estabelecer correlações e a levantar hipóteses, podendo também ser usados nas interpretações dos fenômenos naturais e previsões comportamentais de sustentabilidade de florestas para refletir a trajetória e a perpetuação dos ecossistemas (PIÑA-RODRIGUES et al., 2015).

Uma vez que as interações na restauração ocorrem em diferentes níveis, a seleção multicriterial dos indicadores empregando o método MESMIS torna possível integrar e utilizar as variações temporais e 
espaciais combinadas aos processos ecológicos que se deseja analisar (MENDOZA; PRABHU, 2003). Em função disso, o protocolo de indicadores empregado baseou-se no método MESMIS (MASERA; ASTIER; LÓPEZ-RIDAURA, 1999) e foi adaptado por Piña-Rodrigues et al. (2015) (Tabela 1). Para os atributos de estabilidade, resiliência e confiabilidade foram definidos descritores e cenários referenciais avaliados por meio de indicadores e seus parâmetros. Para cada indicador foram propostos cenários e referenciais, positivos e negativos, baseados em revisão bibliográfica (Tabela 1). A seguir foram atribuídas notas variando

Tabela 1 - Protocolo de avaliação da funcionalidade ecológica de áreas de restauração empregado com base nos atributos de estabilidade, resiliência e confiabilidade do sistema baseado no método MESMIS.

\begin{tabular}{|c|c|c|c|c|}
\hline$\frac{8}{\frac{2}{3}}$ & 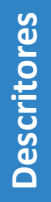 & Indicadores & Cenários e referenciais & Parâmetros \\
\hline \multirow{5}{*}{ 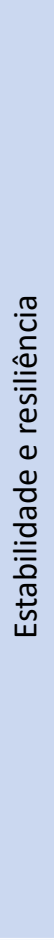 } & \multirow{5}{*}{ 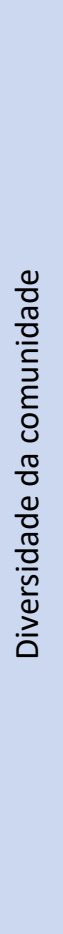 } & $\begin{array}{l}\text { Diversidade de espécies } \\
\text { arbóreas }\left(\mathrm{H}^{\prime}\right)\end{array}$ & $\begin{array}{l}\text { Índice de Shannon próximo ao } \\
\text { esperado para fragmentos referência } \\
\text { estudados da região com } \mathrm{H}^{\prime}=3,676 \text {. }\end{array}$ & $\begin{array}{c}H^{\prime}>3,0=3 \\
1,0<H^{\prime}<2,9=2 \\
H^{\prime}<0,9=1\end{array}$ \\
\hline & & $\begin{array}{l}\text { Riqueza de espécies } \\
\text { nativas (S) }\end{array}$ & $\begin{array}{l}\text { Indesejável: inferior ao previsto na } \\
\text { Resolução SMA no 08/08. } \\
\text { Regular: baixa diversidade prejudica o estabelecimento da } \\
\text { comunidade futura. } \\
\text { Desejável: de acordo com a Resolução SMA no } 08 / 08 \text {. }\end{array}$ & $\begin{array}{c}\text { № espécies }>30=3 \\
10>\text { № espécies }<30=2 \\
\text { № espécies }<10=1\end{array}$ \\
\hline & & $\begin{array}{l}\text { Densidade de indivíduos } \\
\text { arbóreos }\left(\mathrm{n}^{\circ} \cdot \mathrm{ha}^{-1}\right)(\mathrm{d})\end{array}$ & $\begin{array}{l}\text { Indesejável: alta mortalidade, considerando a densidade de } \\
\text { plantas recomendada pela Resolução SMA no 08/08. } \\
\text { Regular: valores médios de densidade baseados na Resolução } \\
\text { SMA no 08/08. } \\
\text { Desejável: valores aproximados aos recomendados pela } \\
\text { Resolução SMA no 08/08. }\end{array}$ & $\begin{array}{c}>1.200=3 \\
>800 \text { e }<1.200=2 \\
>400 \text { e }<800=1 \\
<400=0\end{array}$ \\
\hline & & Equitabilidade (J') & $\begin{array}{c}\text { Índice de Pielou (J') similar ao de áreas } \\
\text { de floresta secundária da região. Valor da } \\
\left.\text { área de referência ( } \mathrm{J}^{\prime}=0,904\right) \text {. }\end{array}$ & $\begin{array}{c}\mathrm{J}^{\prime} \geq 1-\text { alta }=3 \\
0,5<J^{\prime}<0,9-\text { média }=2 \\
\mathrm{~J}^{\prime}<0,5-\text { baixa }=1\end{array}$ \\
\hline & & $\begin{array}{l}\text { № de indivíduos/grupo } \\
\text { sucessional (IND/Ge) }\end{array}$ & $\begin{array}{c}\text { Indesejável: não atende à Resolução SMA no 08/08. } \\
\text { Desejável: atende à Resolução SMA no 08/08. }\end{array}$ & $\begin{array}{c}>40 \% \text { e }<60 \% \text { de } \\
\text { espécies/grupo }=3 \\
\text { IND }<40 \% \text { e IND }>60 \% \\
\text { de espécies/grupo }=1\end{array}$ \\
\hline \multirow{3}{*}{ 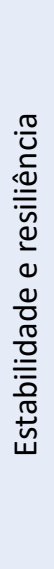 } & \multirow{3}{*}{ 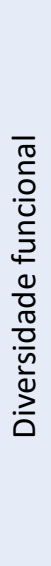 } & $\begin{array}{l}\text { № de espécies por grupos } \\
\text { sucessionais das espécies } \\
\text { arbóreas } \mathrm{D}(\mathrm{GE})\end{array}$ & $\begin{array}{c}\text { Maior número de espécies não pioneiras } \\
\text { presentes no sistema. }\end{array}$ & $\begin{array}{l}P<N P=3 \\
P \pm N P=2 \\
P>N P=1\end{array}$ \\
\hline & & $\begin{array}{l}\text { Altura média dos } \\
\text { indivíduos arbóreos }(\mathrm{m})- \\
\text { incremento médio anual } \\
\text { (IMA) }\end{array}$ & $\begin{array}{c}\text { Indesejável: reflete o crescimento lento dos indivíduos ou } \\
\text { replantios constantes (IMA }<0,5 \mathrm{~m} \text { ). } \\
\text { Regular: valores considerados médios de crescimentos para } \\
\text { plantios (IMA de } 0,5 \text { a } 1,0 \mathrm{~m} \text { ) } \\
\text { Desejável: valores considerados compatíveis com plantios de } \\
\text { restauração (IMA }>1,5 \mathrm{~m} \text { ). } \\
\text { Referencial: MELO e DURIGAN (2007). }\end{array}$ & $\begin{array}{c}\text { IMA }>2,0=3 \\
1<\mathrm{IMA}<2,0=2 \\
0,5<\mathrm{IMA}<1,0=1 \\
\mathrm{IMA}<0,5=0\end{array}$ \\
\hline & & $\begin{array}{l}\text { Área basal média - AB } \\
\qquad\left(\mathrm{m}^{2}\right)\end{array}$ & Similar à AR & $\begin{array}{l}A B>A R=3 \\
A B \approx A R=2 \\
A B<A R=1\end{array}$ \\
\hline
\end{tabular}




\section{Tabela 1 - Continuação}

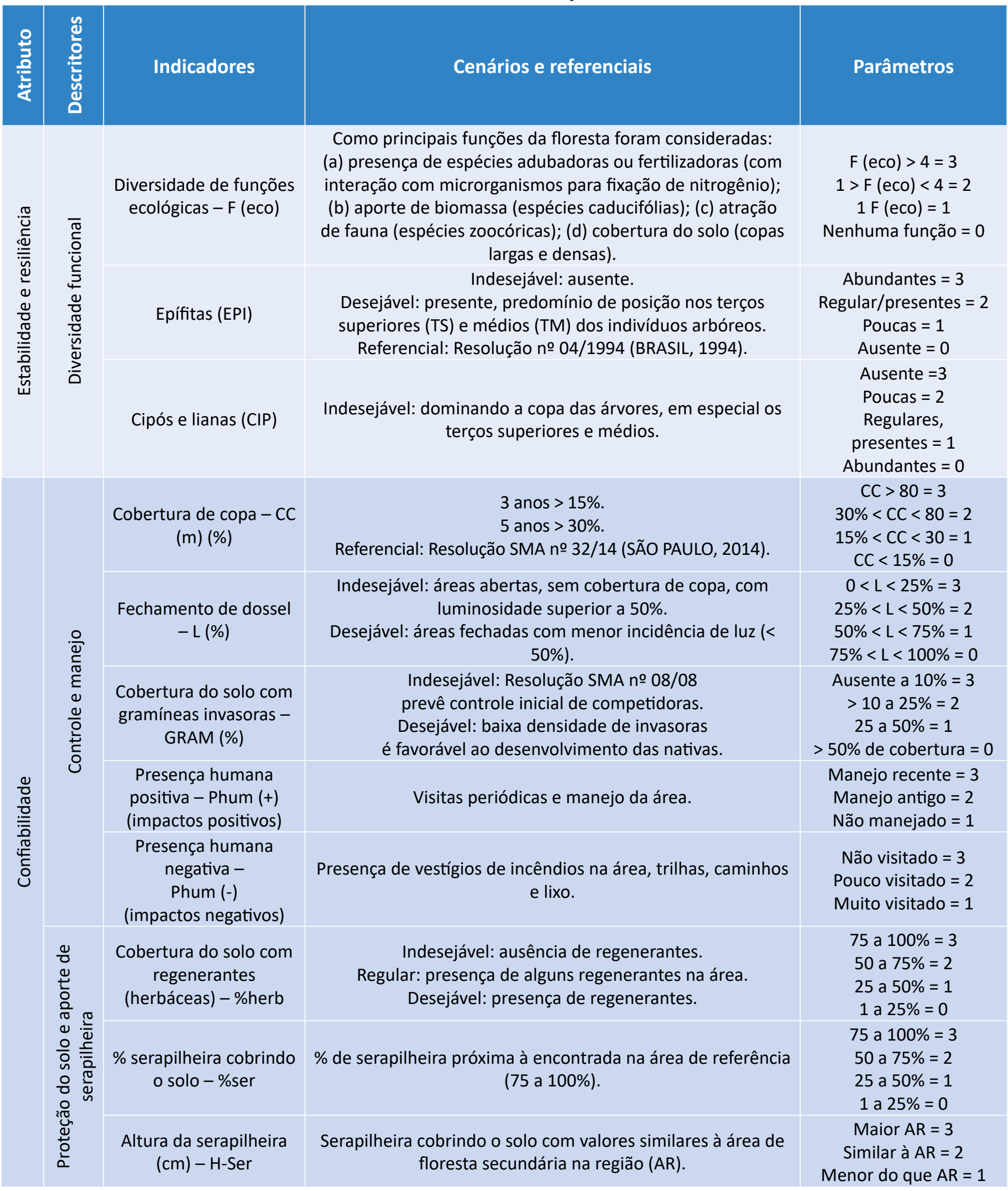

Fonte: MESMIS (MASERA; ASTIER; LÓPEZ-RIDAURA, 1999); descritores, indicadores, cenários e referenciais e parâmetros adaptados por Piña-Rodrigues et al. (2015). 
de 0 a 1 (grau crítico - ruim, inexistente ou distinto do cenário positivo), 2 (grau aceitável) e 3 (grau desejado de sustentabilidade, similar ao cenário positivo). A partir das notas obtidas, foram elaborados gráficos de radar, contemplando os indicadores de estabilidade, resiliência e confiabilidade. Na sua interpretação, cada raio do gráfico representa um dos indicadores,

\subsection{Análise da estrutura e diversidade vegetal}

A área basal média e a densidade de indivíduos foram estimadas por hectare. Para o cálculo da área basal da parcela, soma-se a área basal dos troncos das árvores amostradas. A área basal de uma árvore foi calculada pela Equação 1.

$A B=\pi r^{2}$

Em que:

$A B=$ área basal $\left(m^{2}\right)$;

$\pi$ = razão entre perímetro e diâmetro de uma circunferência $(\cong 3,1416)$;

$r=$ raio da circunferência a altura do colo do indivíduo arbóreo.

Após o cálculo da área basal de cada parcela, foi calculada a média entre parcelas para se obter a área basal média. Posteriormente foi estimada a área basal média por ha através da Equação 2.

\section{Análise de dados}

Os dados foram avaliados quanto à normalidade por meio do teste de Shapiro-Wilk, e a análise de variância (ANOVA) foi realizada. Com base nos resultados do teste de normalidade e da análise de variância, as áreas foram comparadas entre si pelo teste de Tukey $(p<0,05)$. Todas as análises foram realizadas com o programa Past 3.13 (HAMMER; HARPER; RYAN, 2001). Para o conjunto de indicadores de diversidade da co- sendo seu comprimento proporcional à nota do indicador (0 a 3). Uma linha é desenhada ligando os valores de cada raio, permitindo melhor visualização e análise dos indicadores e das condições de cada área de estudo. Esse procedimento permitiu que os dados analisados pudessem ser comparados dentro da mesma unidade de avaliação.

ABha $\frac{A B p \cdot 1000}{A P}$

Em que:

$A B=$ área basal média por hectare $\left(m^{2}\right)$;

$A B p=$ área basal média das parcelas $\left(\mathrm{m}^{2}\right)$;

$A P=$ área da parcela $\left(m^{2}\right)$.

A densidade de indivíduos das parcelas foi calculada pela divisão do número de indivíduos de cada parcela pela área da parcela $\left(\mathrm{m}^{2}\right)$. 0 incremento médio anual (IMA) foi calculado para cada modelo, dividindo-se a altura média dos indivíduos arbóreos amostrados em cada área pela idade, em anos. A diversidade de espécies foi estimada pelo índice de Shannon-Wiever $\left(H^{\prime}\right)$, a equitabilidade por Pielou ( $\mathrm{J}^{\prime}$ ) e a riqueza (S) segundo Magurran (2004).

munidade e funcional, controle e manejo, proteção do solo e aporte de serapilheira foi calculado o índice de consolidação da funcionalidade ecológica (ICFE), obtido pela Equação 3.

$$
\text { ICFE } \frac{\text { Snotas dos indicadores no de indicadores }}{(n \text { - de indicadores }) \bullet(n-\text { de parâmetros por indicador })}
$$

\section{RESULTADOS E DISCUSSÃO}

Com a análise de variância (ANOVA) ficou evidenciada a diferença significativa de diversidade entre a áreas $\left(F=8,909 ; w^{2}=0,5826 ; p=0,001806\right)$, o teste de Tukey apontou que DDF e P\&D-35 não diferiram entre si, mas sim das demais áreas. Essa diferença de diversidade entre as áreas está relacionada à riqueza de espécies encontrada nos modelos DDF e P\&D-35, que foi mais que o dobro da observada nas demais áreas 
(Tabela 2). Deve-se destacar que o índice de diversidade de Shannon-Weaver $\left(\mathrm{H}^{\prime}\right)$ considera, além da riqueza de espécies (S), a uniformidade de distribuição dos indivíduos entre as espécies. Assim, embora AR e P\&D-60 tenham riqueza de espécies menor do que DDF e P\&D-35, essas apresentaram distribuição equilibrada de indivíduos entre as espécies. Isso foi confirmado pelo índice de Pielou (J') (Tabela 2), o qual não

Tabela 2 - Valores obtidos para os indicadores dos atributos de estabilidade, resiliência e confiabilidade das áreas de restauração estudadas.

\begin{tabular}{|c|c|c|c|c|c|}
\hline Atributos & Indicadores & AR & DDF & P\&D-35 & P\&D-60 \\
\hline \multirow{15}{*}{$\begin{array}{l}\text { Estabilidade e } \\
\text { resiliência }\end{array}$} & \multicolumn{5}{|c|}{ Diversidade de espécies } \\
\hline & $\mathrm{H}^{\prime}$ (bits.indv $\left.{ }^{\top}\right)$ & 2,66 & 3,62 & 3,89 & 3,11 \\
\hline & $S(n \circ)$ & 19 & 87 & 75 & 32 \\
\hline & $d(n \circ)$ & 1155 & 18229 & 2156 & 2000 \\
\hline & J & 0,904 & 0.810 & 0,901 & 0,897 \\
\hline & Ind/GE (\%) - NP & 51,0 & 51,0 & 47,8 & 38,3 \\
\hline & Ind/GE (\%) - P & 49,0 & 49,0 & 51,6 & 61,7 \\
\hline & \multicolumn{5}{|c|}{ Diversidade funcional } \\
\hline & $D(G E)(n \circ P)$ & 9 & 34 & 30 & 13 \\
\hline & $D(G E)(n \circ N P)$ & 10 & 53 & 45 & 19 \\
\hline & IMA (m/ano) & - & 0,789 & 0,841 & 0,561 \\
\hline & $A B\left(m^{2}\right)$ ha & 20,93 & 32,06 & 9,50 & 2,55 \\
\hline & $F(e c o)$ & 3 & 4 & 3 & 2 \\
\hline & EPI & 2 & - & - & - \\
\hline & CIPL & 1 & 3 & 3 & 3 \\
\hline \multirow{10}{*}{ Confiabilidade } & \multicolumn{5}{|c|}{ Controle e manejo } \\
\hline & CC (\%) & 80,71 & 82,73 & 26,25 & 20,44 \\
\hline & $L(\%)$ & 6,3 & 0,4 & 64,3 & 69,2 \\
\hline & GRAM (\%) & 19,4 & 10,6 & 67,5 & 83,9 \\
\hline & Phum (+) & 1 & 2 & 2 & 1 \\
\hline & Phum (-) & 1 & 1 & 1 & 2 \\
\hline & \multicolumn{5}{|c|}{ Proteção do solo e aporte de serapilheira } \\
\hline & \%herb & 4,2 & 0,9 & 12,6 & - \\
\hline & $\%$ ser & 91,7 & 97,7 & 12,9 & 8,9 \\
\hline & H-ser & 1,3 & 1,2 & 0,2 & 0,1 \\
\hline
\end{tabular}

AR: área de referência de fragmento florestal; DDF: área de restauração no modelo denso-diverso-funcional; P\&D-35: área de restauração no modelo preenchimento e diversidade, com idade de 35 meses; P\&D-60: área de restauração no modelo preenchimento e diversidade, com idade de 60 meses. $\mathrm{H}^{\prime}$ : índice de diversidade de Shannon; S: riqueza de espécies; d: densidade de indivíduos; Ind/GE: porcentagem de indivíduos/grupo ecológico; J: índice de equitabilidade de Pielou; $\mathrm{D}(\mathrm{Ge})$ : diversidade de grupos ecológicos; IMA: incremento médio anual; $A B$ : área basal; $F(e c o)$ : diversidade de funções ecológicas; Epi: epífitas; CIPL: cipós e lianas; CC: cobertura do solo com copa; L: incidência de luz; GRAM(\%): presença gramíneas invasoras; Phum (-): presença humana negativa; Phum (+): presença humana positiva; \%herb: porcentagem de herbáceas; \%ser: porcentagem de serrapilheira; H-ser: altura da serrapilheira. 
apresentou diferença significativa entre as restaurações P\&D-35, P\&D-60 e AR. Já a área em restauração DDF apresentou diferença significativa $(F=8,674$; $\left.w^{2}=0,5752 ; p=0,002022\right)$ em comparação com as outras áreas plantadas em estudo $(p=0,02147 ; p=$ 0,004744; $p=0,004041$ para AR, P\&D-35 e P\&D-60, respectivamente), evidenciando que, apesar da grande riqueza de espécies, DDF foi a área que apresentou distribuição menos equitativa de indivíduos por espécie. Porém, todos os valores encontrados neste estudo foram próximos à média dos observados em estudos fitossociológicos no estado de São Paulo ( $\mathrm{J}^{\prime}=$ 0,8425) (TANUS et al., 2012; COLMANETTI \& BARBOSA, 2013).

Apesar da distribuição uniforme de indivíduos entre espécies, a densidade de indivíduos foi similar entre as áreas $\left(F=54,81 ; w^{2}=0,9047 ; p=1,232 \times 10^{-7}\right)$, excetuando-se o DDF devido à sua metodologia de implantação com alta densidade de plantas $(p=0,0002012$; $p=0,0002012 ; p=0,0002012$ para AR, P\&D-35 e P\&D60 , respectivamente).

Os resultados obtidos mostraram que as análises isoladas, tanto da riqueza quanto da diversidade de espécies, podem mascarar as diferenças na qualidade das áreas restauradas. Baseado nisso, uma das estratégias adotadas em projetos de restauração tem sido a distribuição uniforme de poucos indivíduos em muitas espécies, o que tende a influenciar o índice de Shannon e favorecer a sua aprovação junto aos órgãos de fiscalização que utilizam esses índices como referenciais (FERNANDES; FREITAS; PIÑA-RODRIGUES, 2017).

Em termos de diversidade da comunidade e funcional, observa-se que já aos 53 meses o modelo DDF foi superior à AR para a maioria dos indicadores (Figura 1), excetuando-se apenas a presença de epífitas e o incremento médio anual, que podem estar relacionados com a idade avançada do fragmento, o mesmo não ocorrendo com as demais restaurações. Ainda em relação à diversidade funcional, a maior densidade de plantas em DDF pode ter gerado os altos valores de área basal encontrados, superiores a outras duas áreas de restauração, com 7 anos de plantio $\left(4,13 \mathrm{~m}^{2} / \mathrm{ha}\right.$ e $\left.4,27 \mathrm{~m}^{2} / \mathrm{ha}\right)$ em um fragmento florestal $\left(27,13 \mathrm{~m}^{2} / \mathrm{ha}\right)$ na floresta estacional em Minas Gerais (MARCUZZO et al., 2014) e a plantios de restauração também de 7 anos e em áreas de floresta estacional, situados no Vale do Paranapanema, com 17,26 $\mathrm{m}^{2}$ /ha (MELO; DURIGAN, 2007).

Assim como a área basal reflete a ocupação do espaço horizontal pela vegetação, o mesmo ocorre com a cobertura de copa, que apontou diferença significativa entre as áreas $\left(F=34,76 ; w^{2}=0,8491 ; p=9,677 \times 10^{-7}\right)$, e AR e DDF não apresentaram diferenças significativas entre si, porém ambas diferiram significativamente das restaurações P\&D-35 ( $p=0,0002066$ e $p=0,0002594)$ e P\&D-60 ( $p=0,0002024$ e $p=0,0002347)$, respectivamente. Áreas em que o solo é coberto pela sombra das copas têm o estabelecimento de gramíneas invasoras dificultado (MELO; REIS; RESENDE, 2010; MARTINS, 2011), como ficou evidenciado na presença de gramíneas invasoras entre as áreas $\left(F=16,49 ; w^{2}=\right.$ 0,$7208 ; p=7,168 \times 10^{-5}$ ), em que AR e DDF tiveram menor incidência de gramíneas invasoras do que P\&D35 e P\&D-60. Considerando que a cobertura de copa apresenta alta correlação com a área basal e a idade da restauração (MELO; DURIGAN 2007), a maior área basal observada em DDF pode ter contribuído para se obter menor incidência de luz e redução na presença de gramíneas invasoras já aos 53 meses, contudo, o mesmo ainda não foi constatado para a restauração aos 60 meses (P\&D-60).

Os indicadores de porcentagem de serapilheira ( $F$ $\left.=57,54 ; w^{2}=0,9041 ; p=4,062 \times 10^{-8}\right)$ e altura de serapilheira $\left(F=27,47 ; w^{2}=0,8152 ; p=3,994 \times 10^{-}\right.$ $\left.{ }^{6}\right)$ também apontaram diferença significativa entre as áreas (Tabela 2). AR e DDF diferiram dos modelos de $P \& D$, porém não houve diferença entre eles. Isso evidencia que DDF teve produção de serapilheira similar ao fragmento em estágio mais avançado de regeneração. A presença de serapilheira em ecossistemas florestais pode ser considerada o principal estoque de matéria orgânica e minerais nessas áreas, desempenhando importantes funções no equilíbrio e dinâmica desses ambientes, (SALGADO et al., 2015; BRASIL et al., 2017), fornecendo condições favoráveis ao solo como proteção e retenção de umidade, gerando condições ao desenvolvimento de organismos que contribuem no processo de decomposição (SCORIZA; CORREIA; SILVA, 2017). A partir dos processos de decomposição da serapilheira é que se dão a devolução e a transferência de matéria orgânica e nutrientes ao solo florestal, fornecendo elementos essenciais para manutenção da vegeta- 
A

Estabilidade e resiliência

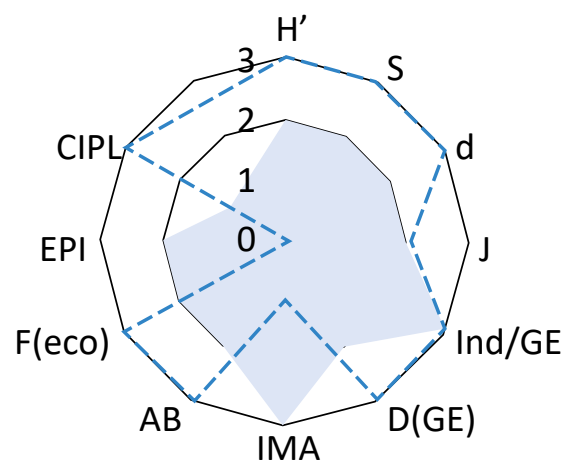

Confiabilidade

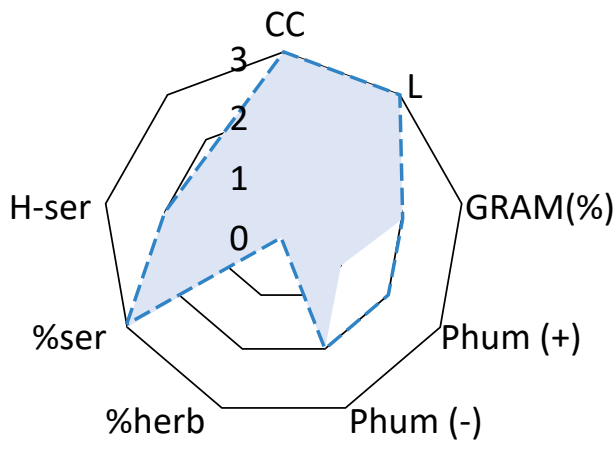

AR ---- DDF

B
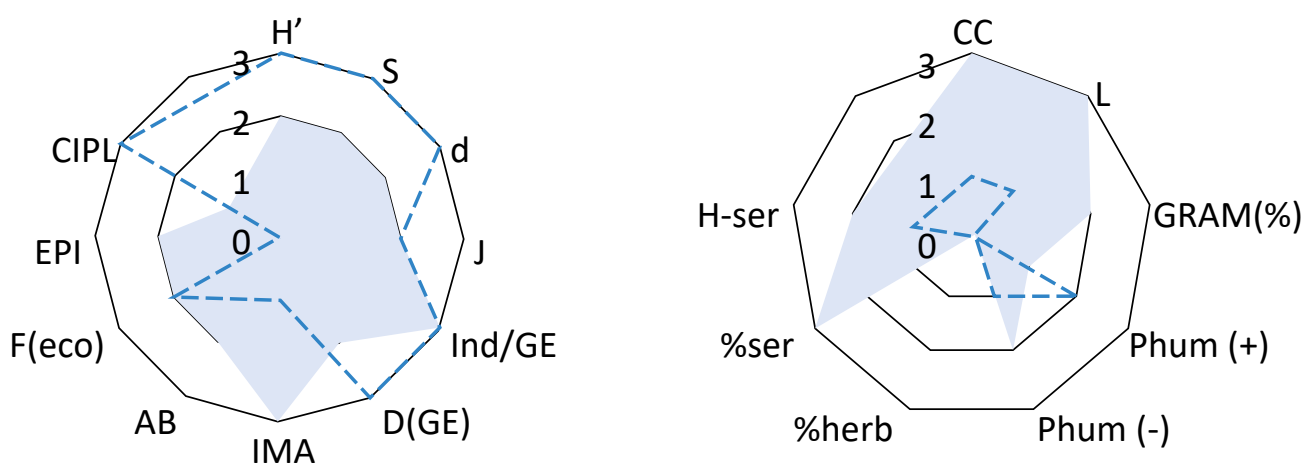

AR

P\&D-35

C
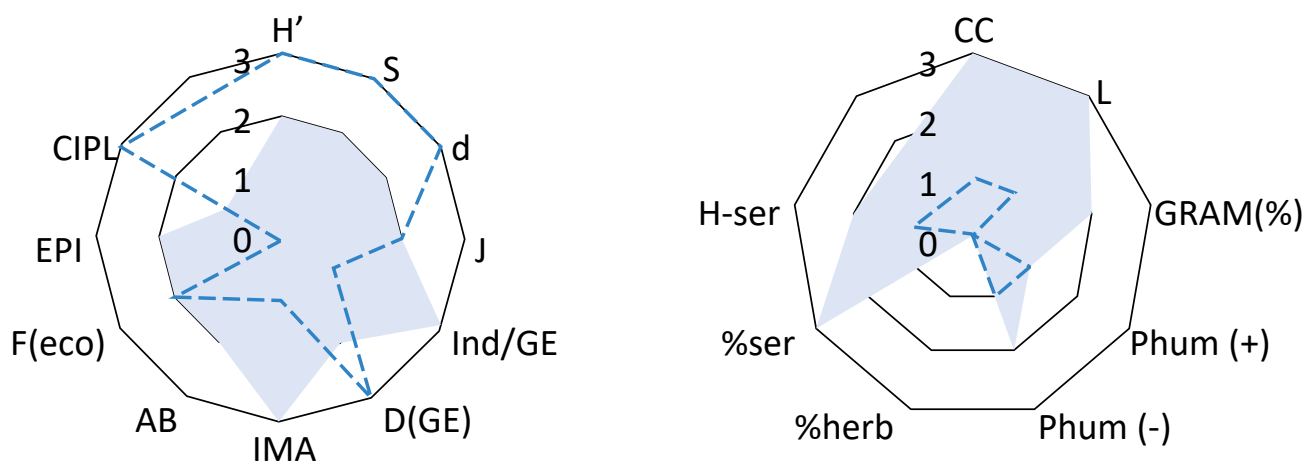

AR $----P \& D-60$

Figura 1 - Gráficos radar, com raios de 0 a 3, conforme os parâmetros da Tabela 1, dos indicadores das áreas de restauração estudadas. (A) área de referência versus modelo denso-diverso-funcional; (B) área de referência versus modelo preenchimento e diversidade 35 meses; (C) área de referência versus modelo preenchimento e diversidade 60 meses. 
ção (VITAL et al., 2004; PAULA; PEREIRA; MENEZES, 2009; DINIZ et al., 2015). Dessa forma, seria plausível supor que em ambientes com bom aporte de serapilheira, como o observado no modelo DDF aos 53 meses, podem estar ocorrendo processos ligados à proteção do solo e à ciclagem de nutrientes.

Na área DDF houve o plantio dominante de espécies não pioneiras ( $53 \%$ não pioneiras e $47 \%$ pioneiras) e maior proporção de indivíduos não pioneiros, seguindo os princípios do método que favorece a formação de multiestratos (MIYAWAKI, 1998). Se por um lado esse procedimento difere dos modelos que utilizam maior porcentagem de espécies e indivíduos de pioneiras (RODRIGUES; BRANCALION; ISERNHAGEN, 2009), os resultados dos indicadores de estabilidade, resiliência e confiabilidade (Figura 1) mostraram que, já aos 53 meses, foi constatada a promoção de condições similares, e até superiores, às do fragmento de referência (Figura 1A). Apesar de AR ter maior idade e desenvolvimento, conforme constatado em relação à presença de epífitas, cipós e lianas e a área basal, os resultados obtidos em DDF de riqueza diversidade de espécies e diversidade funcional podem ter contribuído para a similaridade entre as áreas e maior diversidade de funções ecológicas em DDF em relação à AR.

Em P\&D-35 e P\&D-60, nos fatores geradores de estabilidade e resiliência de ambas restaurações, foram constatados valores de estrutura (área basal) e desenvolvimento (presença de epífitas) inferiores à $A R$, contudo, essas condições podem ser atribuídas à diferença de idade entre elas. Por outro lado, os fatores relativos à sustentabilidade ambiental ao longo do tempo (confiabilidade) foram os que mais distinguiram as restaurações P\&D de AR (Figuras 1B e 1C). As condições inferiores dos plantios P\&D em relação aos indicadores de confiabilidade mostram que essas áreas podem não conseguir se sustentar sozinhas ao longo do tempo, caso não ocorra o seu manejo adequado.

O emprego de alta diversidade de espécies e funcional em restauração proporciona maior estabilidade, criando a complementariedade de nichos ecológicos, já que espécies com nichos complementares respondem aos distúrbios de formas diversas, fomentando estabilidade e resiliência do ecossistema, favorecendo a ocorrência dos processos ecológicos (LANARI; COUTINHO, 2010). Nos modelos P\&D, embora apresentem diversidade de espécies similar à DDF, a ausência de processos ligados à produção de serapilheira pode ser atribuída tanto à composição e densidade de espécies quanto ao manejo inadequado das áreas, o que pode facilitar a degradação e o estabelecimento de gramíneas exóticas no sistema (MELO; REIS; RESENDE, 2010). De maneira geral, até os 53 meses o modelo DDF foi eficiente na recuperação das funções ecológicas capazes de gerar estabilidade e resiliência e de manutenção dos processos ecológicos em condições similares a fragmentos em estádio inicial de sucessão, sendo que seu ICFE (Figura 2) foi similar à AR e superior aos constatados nas áreas restauradas no modelo de preenchimento e diversidade, nas condições testadas. Contudo, conforme se constata pelos valores negativos de ICFE obtidos para P\&D-35 e P\&D-60, essas diferenças se devem principalmente a fatores como a capacidade do sistema manter a produtividade em longo prazo, relacionadas com processos como o aporte de serapilheira e a proteção do solo.

Por outro lado, os fatores relacionados à proteção do solo e ao controle e manejo requerem a revisão de modelos como os adotados no P\&D-35 e P\&D-60, que geram e demandam manejo por períodos prolongados, ou seja, essas áreas necessitam de ações contínuas para que consigam manter uma trajetória ecológica desejada, caso contrário apresentam condições que podem levá-las a se tornarem degradadas novamente. Ao passo que o modelo DDF tende a apresentar alto custo de instalação em função da quantidade de mudas empregada, mas reduz a quantidade e o custo de manutenção (PIÑA-RODRIGUES; REIS; MARQUES, 1997). Nesse sentido, deve-se buscar o equilíbrio entre os fatores que proporcionaram a sua funcionalidade ecológica, como a diversidade da comunidade e funcional, e a densidade de plantas.

De maneira geral, o aumento da área basal, do número de espécies não pioneiras ou mesmo da densidade dos indivíduos pode contribuir para a cobertura do solo, fator importante para a confiabilidade do sistema. Por outro lado, a seleção adequada de espécies deve buscar tanto empregar aquelas caducifólias que possam contribuir com o aporte de serapilheira e de nutrientes no sistema, como também procurar incorporar espécies cuja arquitetura de copa e persistência de folhas sejam capazes de promover o sombreamento do solo e a redução da presença de gramíneas invasoras. 


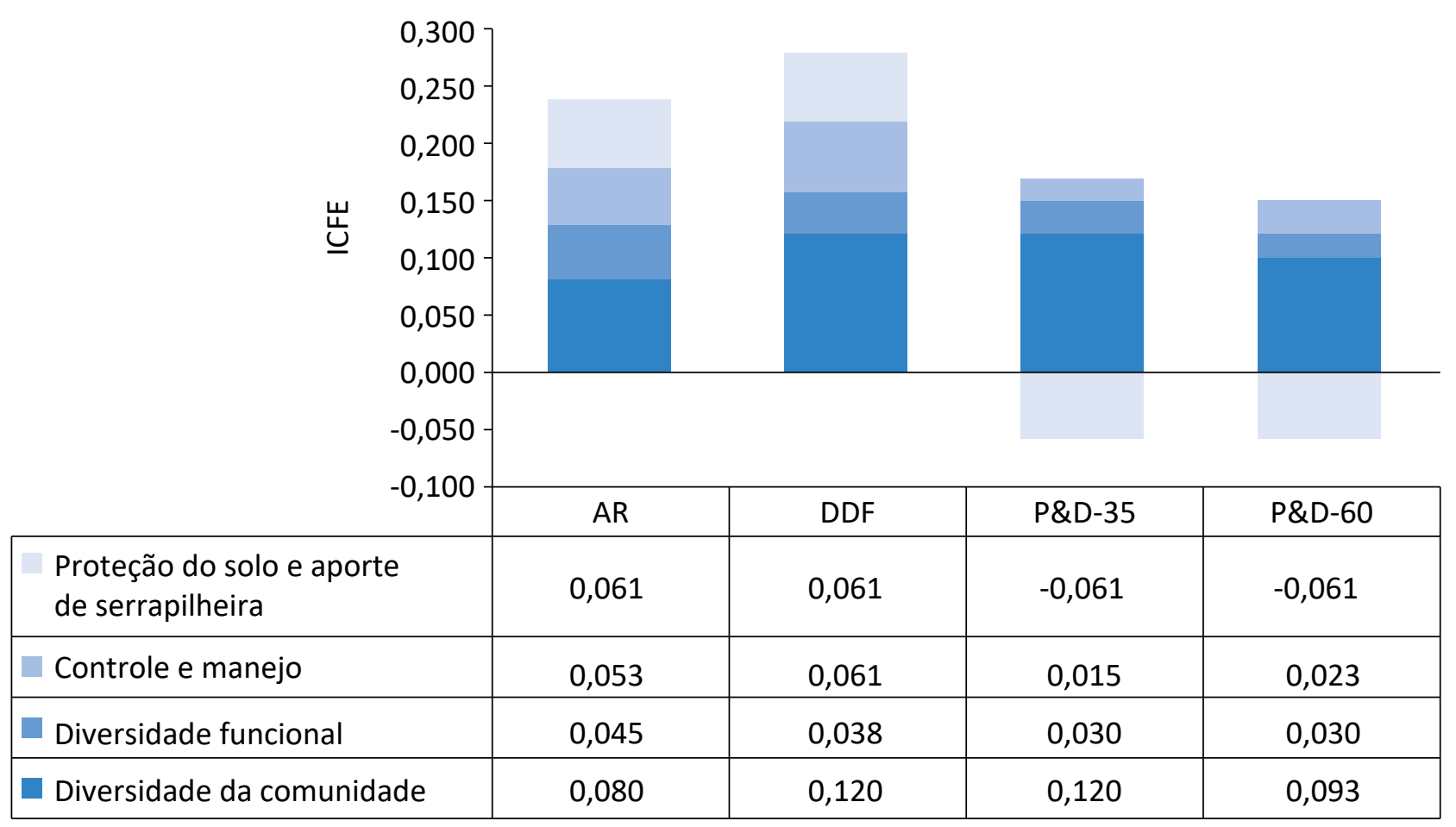

ICFE: índice de consolidação da funcionalidade ecológica; AR: área referência de fragmentos florestais; DDF: sistema de plantio denso-diverso-funcional; P\&D-35: método diversidade e preenchimento com 35 meses de idade; P\&D-60: método diversidade e preenchimento com 60 meses de idade.

Figura 2 - Valores do índice de consolidação da funcionalidade ecológica obtidos para os atributos de estabilidade e resiliência do sistema representados pela diversidade de espécies e funcional e de confiabilidade, representado pela proteção e aporte de serapilheira e o controle e manejo das áreas estudadas na região de Sorocaba, São Paulo.

\section{CONCLUSÕES}

O modelo DDF, baseado em alta densidade de plantas e diversidade de espécies e funcional, foi eficiente na restauração da funcionalidade ecológica já aos 53 meses de idade, diferindo dos modelos P\&D quanto à funcionalidade ecológica em relação às condições para promover a proteção do solo e produção de serapilheira.
Nos modelos P\&D, os fatores referentes à cobertura do solo com copa, incidência de luz, presença de gramíneas invasoras, recobrimento do solo com herbáceas e serapiIheira foram determinantes para que não tenham sido estabelecidas as condições de funcionalidade ecológica capazes de permitir a autossustentabilidade das áreas sem a necessidade de execução de manejo e práticas culturais.

\section{REFERÊNCIAS}

BASTIAN, O. The role of biodiversity in supporting ecosystem services in Natura 2000 sites. Ecological Indicators, v. 24, p. 12-22, jan. 2013. http://dx.doi.org/10.1016/j.ecolind.2012.05.016

BRANCALION, P. H. S.; RODRIGUES, R. R.; GANDOLFI, S.; KAGEYAMA, P. Y.; NAVE, A. G.; GANDARA, F. B.; BARBOSA, L. M.; TABARELLI, M. Instrumentos legais podem contribuir para a restauração de florestas tropicais biodiversas. Revista Árvore, v. 34, n. 3, p. 455-470, 2010. http://dx.doi.org/10.1590/S0100-67622010000300010 
BRASIL. Conselho Nacional do Meio Ambiente. Resolução no 4, de 4 de maio de 1994. Define vegetação primária e secundária nos estágios inicial, médio e avançado de regeneração da mata atlântica. Brasil, 1994. Disponível em: <http://www.mma.gov.br/port/conama/res/res94/res0494.html>. Acesso em: jul. 2016.

BRASIL, J. B.; ANDRADE, E. M.; NASCIMENTO AQUINO, D.; PEREIRA JÚNIOR, L. R. Sazonalidade na produção de serrapilheira em dois manejos no semiárido tropical. JEAP, v. 2, n. 3, p. 167-176, 2017. https://doi.org/10.24221/ jeap.2.3.2017.1335.167-176

CADOTTE, M. W.; CARSCADDEN, K.; MIROTCHNICK, N. Beyond species: functional diversity and the maintenance of ecological processes and services. Journal of Applied Ecology, v. 48, p. 1079-1087, 2011. https://doi.org/10.1111/ j.1365-2664.2011.02048.x

CARDINALE, B. J.; SRIVASTAVA, D. S.; DUFFY, J. E.; WRIGHT, J. P.; DOWNING, A. L.; SANKARAN, M.; JOUSEAU, C.; CADOTTE, M. W.; CARROLL, I. T.; WEIS, J. J.; HECTOR, A.; LOREAU, M. Effect of biodiversity on the functioning of ecosystems: a summary of 164 experimental manipulations of species richness. Ecology, v. 90, n. 3, p. 854-854, mar. 2009.

CIANCIARUSO, M. V.; SILVA, I. A.; BATALHA, M. A. Diversidades filogenética e funcional: novas abordagens para a Ecologia de comunidades. Biota Neotropica, v. 9, n. 3, p. 93-103, jun. 2009. http://dx.doi.org/10.1590/S1676-06032009000300008

COLMANETTI, M. A. A.;BARBOSA, L. M. Fitossociologia e estrutura doestratoarbóreo de um reflorestamento com espécies nativas em Mogi-Guaçu, SP, Brasil. Hoehnea, v. 40, n. 3, p. 419-435, ago. 2013. http://dx.doi.org/10.1590/S2236-89062013000300003

DEAN, W. A ferro e fogo: a história e a devastação da Floresta Atlântica brasileira. São Paulo: Companhia das Letras, 1996. 504 p.

DÍAZ, S.; CABIDO, M. Vive la differénce: plant functional diversity matters to ecosystem processes. Trends in Ecology \& Evolution, v. 16, n. 11, p. 646-655, nov. 2001. https://doi.org/10.1016/S0169-5347(01)02283-2

DINIZ, A. R.; MACHADO, D. L.; PEREIRA, M. G.; BALIEIRO, F. C.; MENEZES, C. E. G. Biomassa, estoques de carbono e de nutrientes em estádios sucessionais da Floresta Atlântica, RJ. Revista Brasileira de Ciências Agrárias, v. 10, n. 3, p. 443451, 2015. https://doi.org/10.5039/agraria.v10i3a4264

DURIGAN, G.; ENGEL, V. L.; TOREZAN, J. M.; MELO, A. C. G.; MARQUES, M. C. M.; MARTINS, S. V.; REIS, A.; SCARANO, F. R. Normas jurídicas para a restauração ecológica: uma barreira a mais a dificultar o êxito das iniciativas? Revista Árvore, v. 34, n. 3, p. 471-485, 2010.

ENGEL, V. L. Abordagem "BEF": um novo paradigma na restauração de ecossistemas? In: SIMPÓSIO DE RESTAURAÇÃO ECOLÓGICA: DESAFIOS ATUAIS E FUTUROS, 4., 2011. Anais... São Paulo, 2011. p. 155-165.

ENGEL, V. L.; PARROTTA, J. A. Definindo a restauração ecológica: tendências e perspectivas mundiais. In: KAGEYAMA, P. Y.; OLIVEIRA, R. E. de; MORAES, L. F. D. de; ENGEL, V. L.; GANDARA, F. B. (Orgs.). Restauração Ecológica de Sistemas Naturais. Botucatu: Ed. FEPAF, 2003. p. 3-25.

FERNANDES, G. E.; FREITAS, N. P.; PIÑA-RODRIGUES, F. C. M. Cobertura florestal ou função ecológica: a eficácia da restauração na bacia do rio Sorocaba e médio tietê. Revista Brasileira de Ciências Ambientais, v. 44, p. 127-145, jun. 2017. https://doi.org/10.5327/Z2176-947820170184

FORRESTER, D. I.; BAUHUS, J. A review of processes behind diversity-productivity relationships in forests. Current Forestry Reports, v. 2, n. 1, p. 45-61, 2016. https://doi.org/10.1007/s40725-016-0031-2

GANDOLFI, S.; BELOTTO, A.; RODRIGUES, R. R. Inserção do conceito de grupos funcionais na restauração, baseada no conhecimento da biologia das espécies. In: RODRIGUES, R. R.; BRANCALION, P. H. S.; ISERNHAGEN, I. Pacto pela restauração da Floresta Atlântica: referencial dos conceitos e ações de restauração. São Paulo: Instituto BioAtlântica, 2009. 256 p.

HAMMER, O.; HARPER, D. A. T.; RYAN, P. D. Past: Paleontological Statistics Software Pack-age for Education and Data Analysis. Palaeontologia Electronica, n. 4, p. 1-9, 2001. 
HOOPER, D. U.; CHAPIN, F. S.; EWEL, J. J.; HECTOR A.; INCHAUSTI, P.; LAVOREL, S.; LAWTON, J. H.; LODGE, D. M.; LOREAU, M.; NAEEM, S.; SCHMID, B.; SETALA, H.; SYMSTAD, A. J.; VANDERMEER, J.; WARDLE, D. A. Effects of biodiversity on ecosystem functioning: a consensus of current knowledge. Ecological Monographs, n. 75, v. 1, p. 3-35, fev. 2005. https://doi.org/10.1890/04-0922

INSTITUTO NACIONAL DE METEOROLOGIA (INMET). Dados climáticos da Estação de Sorocaba: série histórica de 2015. Banco de dados do Instituto Nacional de Meteorologia. Brasil: INMET, 2016. Disponível em: <http://www.inmet.gov. br/>. Acesso em: jun. 2016.

KORTZ, A. R.; COELHO, S.; CASTELLO, A. C. D.; LEITE, E. C.; CORRÊA, L. S.; KOCH, I. Wood vegetation in Atlantic rain forest remnants in Sorocaba (São Paulo, Brazil). Check List, v. 10, n. 2, p. 344, maio 2014. http://dx.doi.org/10.15560/10.2.344

LANARI, M. O.; COUTINHO, R. Biodiversidade e Funcionamento de Ecossistemas: síntese de um paradigma e sua expansão em ambientes marinhos. Oecologia Australis, v. 14, n. 4, p. 959-988, dez. 2010. http://dx.doi.org/10.4257/ oeco.2010.1404.09

LAUGHLIN, D. C. Applying trait-based models to achieve functional targets for theory-driven ecological restoration. Ecology Letters, v. 17, n. 7, p. 771-784, 2014. https://doi.org/10.1111/ele.12288

LELES, P. S. S.; ABURRE, G. W.; ALONSO, J. M.; NASCIMENTO, D. F.; LISBOA, A. C. Crescimento de espécies arbóreas sob diferentes espaçamentos em plantio de recomposição florestal. Scientia Forestalis, v. 39, n. 90, p. 231-239, 2011.

LOREAU, M. Biodiversity and ecosystem functioning: a mechanistic model. Proceedings of the National Academy of Sciences of the United States of America, v. 95, n. 10, p. 5632-5636, 1998.

MAGURRAN, A. E. Measuring biological diversity. Oxford: Blackwell Science, 2004. 256 p.

MARCUZZO, S. B.; ARAÚJO, M. M.; RORATO, D. G.; MACHADO, J. Comparação entre áreas em restauração e área de referência no Rio Grande do Sul, Brasil. Revista Árvore, v. 38, n. 6, p. 961-972, 2014. http://dx.doi.org/10.1590/S010067622014000600001

MARTINS, A. F. Controle de gramíneas exóticas invasoras em área de restauração ecológica com plantio total, Floresta Estacional Semidecidual, Itu, SP. 112f. Dissertação (Mestrado em Recursos Florestais) - Universidade de São Paulo, Piracicaba, 2011.

MASERA, O.; ASTIER, M.; LÓPEZ-RIDAURA, S. Sustentabilidad y Manejo de Recursos Naturales: el marco de evaluación MESMIS. México: Mundi-Prensa, 1999.

MELO, A. C. G.; DURIGAN, G. Evolução estrutural de reflorestamentos de restauração de matas ciliares no Médio Vale do Paranapanema. Scientia Forestalis, v. 73, p. 101-111, mar. 2007.

MELO, A. C. G.; REIS, C. M.; RESENDE, R. U. Guia para monitoramento de reflorestamentos para restauração. Circular Técnica, São Paulo, v. 1, jan. 2010. Disponível em: <http://www.sigam.ambiente.sp.gov.br/sigam3/Repositorio/222/ Documentos/2010_Workshop\%20monitoramento\%20areas\%20em\%20recuperacao/Guia\%20para\%20 monitoramento\%20de\%20reflorestamentos\%20para\%20restauracao.pdf>. Acesso em: 8 jul. 2016.

MENDOZA, G. A.; PRABHU, R. Qualitative multi-criteria approaches to assessing indicators of sustainable forest resource management. Forest Ecology and Management, v. 174, n. 1-3, p. 329-343, 2003. http://dx.doi.org/10.1016/ S0378-1127(02)00044-0

MIYAWAKI, A. Creative ecology: restoration of native forests by native trees. Plant Biotechnology, v. 16, p. 15-25, 1999.

. Restoration of urban green environments based on the theories of vegetation ecology. Ecological Engineering, v. 11, n. 1-4, p. 157-165, 1998. https://doi.org/10.1016/S0925-8574(98)00033-0 
MIYAWAKI, A.; ABE, S. Public awareness generation for the reforestation in Amazon tropical lowland region. Tropical Ecology, v. 45, n. 1, p. 59-65, 2004.

NAEEM, S. Disentangling the impacts of diversity on ecosystem functioning in combinatorial experiments. Ecology, v. 83, p. 2925-2935, 2002. https://doi.org/10.2307/3072027

. Species redundancy and ecosystem reliability. Conservation Biology, v. 12, p. 39-45, fev. 1998. https://doi. org/10.1111/j.1523-1739.1998.96379.x

OLIVER, T. H.; HEARD, M. S.; ISAAC, N. J. B.; ROY, D. B.; PROCTER, D.; EIGENBROD, F.; FRECKLETON, R.; HECTOR, A.; ORME, C. D. L.; PETCHEY, O. L.; PROENÇA, V.; RAFFAELLI, D.; SUTTLE, K. B. A synthesis is emerging between biodiversityecosystem function and ecological resilience research: reply to Mori. Trends in Ecology \& Evolution, v. 31, n. 2, p. 89-92, 2016. https://doi.org/10.1016/j.tree.2015.12.008

PAULA, R. R.; PEREIRA, M. G.; MENEZES, L. F. T. Aporte de nutrientes e decomposição da serapilheira em três fragmentos florestais periodicamente inundados na ilha da Marambaia, RJ. Ciência Florestal, v. 19, n. 2, p. 139-148, 2009. http:// dx.doi.org/10.5902/19805098405

PETCHEY, O. L.; GASTON, K. J. Functional diversity: back to basics and looking forward. Ecological Letters, v. 9, n. 6, p. 741-758, jun. 2006. https://doi.org/10.1111/j.1461-0248.2006.00924.x

PETCHEY, O. L.; HECTOR, A.; GASTON, K. J. How do different measures of functional diversity perform? Ecology, v. 85, n. 3, p. 847-857, mar. 2004. https://doi.org/10.1890/03-0226

PIÑA-RODRIGUES, F. C. M.; REIS, L. L.; MARQUES, S. S. Sistema de plantio adensado para a revegetação de áreas degradadas da Floresta Atlântica: bases ecológicas e comparações de custo/benefício com o sistema tradicional. Floresta e Ambiente, n. 4, p. 30-41, 1997.

PIÑA-RODRIGUES, F. C. M.; SILVA, J. M.; PIOTROWSKI, I.; GALETTI, G.; LOPES, G. R.; FRANCO, F. S.; ALVARES, S. M. R. Protocolo de Monitoramento de Funcionalidade Ecológica de Áreas de Restauração. 2015. Disponível em: <https://www. researchgate.net/publication/283355875_PROTOCOLO_DE_MONITORAMENTO_DA_FUNCIONALIDADE_ECOLOGICA_ DE_AREAS_DE_RESTAURACAO\#share>. Acesso em: jul. 2016. https://doi.org/10.13140/RG.2.1.2324.1681

RODRIGUES, C. M. Eficiência na adoção de medidas para recuperação de áreas degradadas no estado de São Paulo. Dissertação (Mestrado) - Universidade Federal de São Carlos, Sorocaba, 2013.

RODRIGUES, R. R.; BRANCALION, P. H. S.; ISERNHAGEN, I. Pacto pela restauração da Floresta Atlântica. Piracicaba: Instituto Bio Atlântica, 2009. 264 p.

ROSS, J. L. S.; MOROZ, I. C. Mapa Geomorfológico do Estado de São Paulo. São Paulo: Laboratório de Geomorfologia Depto. de Geografia FFLCH-USP/Laboratório de Cartografia Geotécnica, 1997. 63 p.

ROSSI, M. Mapa Pedológico do Estado de São Paulo: revisado e ampliado. São Paulo: Instituto Florestal, 2017. v. $1.118 \mathrm{p}$.

SALGADO, E. V.; ANDRADE, E. M.; HEVIA, J. N.; NUNES, E. P.; RODRIGUES, M. M. A. Rainfall patterns and the contribution of litter in the caatinga dry tropical forest. Revista Ciência Agronômica, v. 46, p. 299-309, 2015. https:// doi.org/10.5935/1806-6690.20150009

SÃO PAULO. Secretaria de Meio Ambiente do Estado de São Paulo. Resolução no 8/2008, de 31 de janeiro de 2008. Diário Oficial do Estado de São Paulo, Seção I, 2008.

. Secretaria de Meio Ambiente do Estado de São Paulo. Resolução no 32/2014, de 5 de abril de 2014. Diário Oficial do Estado de São Paulo, Seção I, p. 36-37, 2014. 
SCHEFFER, M.; CARPENTER, S. R.; DAKOS, V.; VAN NES, E. H. Generic indicators of ecological resilience: inferring the chance of a critical transition. Annual Review of Ecology, Evolution, and Systematics, v. 46, p. 145-167, 2015. https:// doi.org/10.1146/annurev-ecolsys-112414-054242

SCHIRONE, B.; SALIS, A.; VESSELLA, F. Effectiveness of the Miyawaki method in Mediterranean forest restoration programs. Landscape and Ecological Engineering, v. 7, n. 1, p. 81-92, jan. 2011. https://doi.org/10.1007/s11355-010-0117-0

SCORIZA, R. N.; CORREIA, M. E. F.; SILVA, E. M. R. O estoque de serrapilheira é eficiente como indicador ambiental em fragmentos florestais de encosta? Revista Brasileira de Ciências Agrárias, v. 12, n. 1, p. 79-85, 2017. https://doi. org/10.5039/agraria.v12i1a5418

SOARES-FILHO, B.; RAJÃO, R.; MACEDO, M.; CARNEIRO, A.; COSTA, W.; COE, M.; RODRIGUES, H.; ALENCAR, A. Cracking Brazil's forest code. Science, v. 344, n. 6182, p. 363-364, 2014. https://doi.org/10.1126/science.1246663

SOCIETY FOR ECOLOGICAL RESTORATION INTERNATIONAL SCIENCE (SER); POLICY WORKING GROUP. The SER International Primer on Ecological Restoration. 2004. Disponível em: <http://www.ser.org>. Acesso em: maio 2016.

SUDDING, K. N.; GROSS, K. L. The dynamic nature of ecological systems: multiple statesand restoration trajectories. In: FALK, D. A.; PALMER, M. A.; ZEDLER, J. B. (Orgs.). Foundations of Restoration Ecology. Washington, D.C.: Island Press, 2006. p. 190-209.

SUGANUMA, M. S.; DURIGAN, G. Indicators of restoration success in riparian tropical forests using multiple reference ecosystems. Restoration Ecology, v. 23, n. 3, p. 238-251, 2015. https://doi.org/10.1111/rec.12168

TANUS, M. R.; PASTORE, M.; BIANCHINI, R. S.; GOMES, E. P. C. Estrutura e composição de um trecho de Mata Atlântica no Parque Estadual das Fontes do Ipiranga, São Paulo, SP, Brasil. Hoehnea, v. 39, n. 1, p. 157-168, mar. 2012. http:// dx.doi.org/10.1590/S2236-89062012000100010

TILMAN, D.; ISBELL, F.; COWLES, J. M. Biodiversity and ecosystem functioning. Annual Review of Ecology, Evolution, and Systematics, v. 45, 2014. https://doi.org/10.1146/annurev-ecolsys-120213-091917

TRINDADE-FILHO, J.; LOYOLA, R. D. O uso de grupos indicadores como atalho para a conservação da biodiversidade. Revista de Biologia Neotropical, v. 7, n. 2, p. 27-38, dez. 2010. https://doi.org/10.5216/rbn.v7i2.15457

VITAL, A. R. T.; GUERRINI, I. A.; FRANKEN, W. K.; FONSECA, R. C. B. Produção de serapilheira e ciclagem de nutrientes de uma floresta estacional semidecidual em zona ripária. Revista Árvore, v. 28, n .6, p. 793-800, 2004. http://dx.doi. org/10.1590/S0100-67622004000600004

WRIGHT, J.; SYMSTAD, A.; BULLOCK, J.; ENGELHARDT, K. M.; JACKSON, L.; BEMHARD, E. Restoring biodiversity and ecosystem function: will an integrated approach improve results? In: NAEEM, D. E.; BUNKER, D. E.; HECTOR, A.; LOREAU, M.; PERRINGS, C. (Orgs.). Biodiversity, ecosystem functioning and human wellbeing. Oxford: Oxford University Press, 2009. p. 167-177. 